PROCEEDINGS OF THE

AMERICAN MATHEMATICAL SOCIETY

Volume 136, Number 10, October 2008, Pages 3701-3707

S 0002-9939(08)09414-8

Article electronically published on May 15, 2008

\title{
MEANS ON CHAINABLE CONTINUA
}

\author{
MIROSŁAW SOBOLEWSKI \\ (Communicated by Alexander N. Dranishnikov)
}

\begin{abstract}
By a mean on a space $X$ we understand a mapping $\mu: X \times X \rightarrow$ $X$ such that $\mu(x, y)=\mu(y, x)$ and $\mu(x, x)=x$ for $x, y \in X$. A chainable continuum is a metric compact connected space which admits an $\varepsilon$ - mapping onto the interval $[0,1]$ for every number $\varepsilon>0$. We show that every chainable continuum that admits a mean is homeomorphic to the interval. In this way we answer a question by P. Bacon. We answer some other questions concerning means as well.
\end{abstract}

A continuum is a metric compact connected space. A continuum $X$ is chainable if for every number $\varepsilon>0$ there exists an $\varepsilon$-mapping of $X$ onto an interval. This is equivalent to the existence of a representation of $X$ as an inverse sequence of $\operatorname{arcs}$ (the joining mappings may be assumed to be surjective). A mapping $f$ of a continuum $X$ onto a continuum $Y$ is said to be weakly confluent if for every continuum $Z \subset Y$ there exists continuum $W \subset X$ such that $f(W)=Z$. A mapping $\mu: X \times X \rightarrow X$, where $X$ is a space, is called a mean on $X$ if for every $x, y \in X$ we have $\mu(x, y)=\mu(y, x)$, and $\mu(x, x)=x$. Here cohomology means the AlexanderCech cohomology. In [2] P. Bacon showed that the sin $\frac{1}{x}$-curve, one of the standard examples of chainable continua, does not admit a mean and asked whether the only chainable continuum with a mean is the arc. Many works give a partial (positive) answer to the problem, for example [1]. A survey of these results can be found in [4]. In this paper we give a complete answer to this problem. Our argument uses the idea of K. Sigmon, who in 7] applied Alexander-Cech cohomology for investigations of compact spaces admitting a mean. On the other hand our proof is similar to the proof that the only pseudo-contractible (in the sense of W. Kuperberg) chainable continuum is an arc in [8].

These results were presented at Henryk Toruńczyk's Seminar at Warsaw University in October 2005. Recently I obtained a preprint by Alejandro Illanes and Hugo Villanueva, who independently solved the problem of P. Bacon by different methods.

Let us recall the following standard fact (see [4]).

Theorem 1. Let $X$ and $Y$ be spaces with means $\mu_{X}$ and $\mu_{Y}$, respectively. Then the formula $\mu\left((x, y),\left(x^{\prime}, y^{\prime}\right)\right)=\left(\mu_{X}\left(x, x^{\prime}\right), \mu_{Y}\left(y, y^{\prime}\right)\right)$ for $(x, y),\left(x^{\prime}, y^{\prime}\right) \in X \times Y$ defines a mean on $X \times Y$.

Received by the editors September 22, 2006, and, in revised form, August 14, 2007.

2000 Mathematics Subject Classification. Primary 54F15.

Key words and phrases. Continuum, chainable, mean.

(C)2008 American Mathematical Society

Reverts to public domain 28 years from publication 
Proof. We have $\mu\left((x, y)\left(x^{\prime}, y^{\prime}\right)\right)=\left(\mu_{X}\left(x, x^{\prime}\right), \mu_{Y}\left(y, y^{\prime}\right)\right)=\left(\mu_{X}\left(x^{\prime}, x\right), \mu_{Y}\left(y^{\prime}, y\right)\right)=$ $\mu\left(\left(x^{\prime}, y^{\prime}\right),(x, y)\right)$ and $\mu((x, y)(x, y))=\left(\mu_{X}(x, x), \mu_{Y}(y, y)\right)=(x, y)$.

Definition 1. The mean on $X \times Y$ we described in Theorem 1 will be called a product mean and will be denoted by $\mu_{X \times Y}$.

Proposition 1. If $\mu_{X \times X}$ is the product mean on the cartesian square $X \times X$, then $\mu_{X \times X}(\Delta \times \Delta)=\Delta$, where $\Delta=\{(x, x) \in X \times X$ : for $x \in X\}$.

Proof. Let $(x, x),(y, y) \in \Delta$. Then $\mu_{X \times X}((x, x),(y, y))=\left(\mu_{X}(x, y), \mu_{X}(x, y)\right) \in$ $\Delta$.

Definition 2. Let $\varepsilon$ be a positive number. $\Delta_{\varepsilon}$ denotes the set $\{(x, y) \in X \times X$ : $\rho(x, y) \leq \varepsilon\}$.

The compactness of a continuum $X$ and Proposition 1 imply the following

Proposition 2. If $X$ is a continuum with a mean $\mu_{X}$, then for every number $\varepsilon>0$ we can choose a number $\delta>0$ such that $\mu_{X \times X}\left(\Delta_{\delta} \times \Delta_{\delta}\right) \subseteq \Delta_{\varepsilon}$.

We have the following

Lemma 1 (Long Fold Lemma). Let $X$ be a chainable continuum. If $X$ is not an arc, then there exists a number $\delta>0$ such that for every number $\varepsilon>0$ there exist mappings $p: X \rightarrow T, r: T \rightarrow W$, where $T, W$ are arcs, and a subcontinuum $Y \subset X$ satisfying the following properties:

(i) $\operatorname{diam} Y>\delta$;

(ii) $r p: X \rightarrow W$ is an $\varepsilon$-mapping;

(iii) the sets $L=p(Y)$ and $J=r p(Y)$ are arcs;

(iv) the mapping $r \mid L: L \rightarrow J$ is open and there exist three different subarcs $L_{1}$, $L_{2}, L_{3}$ of $L$ each of which is mapped homeomorphically onto $J$ (Figure 1 is a graph of such a map).

Proof. Every chainable continuum is irreducible 3], and every locally connected continuum is arcwise connected (Mazurkiewicz-Moore Theorem, 6]). Hence, a chainable continuum $X$ which is not an arc is not locally connected. This means that the continuum $X$ contains a sequence of pairwise disjoint nondegenerate continua $K, K_{1}, K_{2}, K_{3}, \ldots$, such that $\lim K_{i}=K(\underline{6}, 6$. $\$ 49$.VI. Th.1). Put $\delta=$ $\frac{\operatorname{diam} K}{10}$. Now let us consider a representation of $X$ as an inverse sequence of copies of the unit interval $X=\lim _{\longleftarrow}\left\{I_{n}, f_{n}^{m}\right\}$, where $f_{n}^{m}$ are surjections. For a given number $\varepsilon>0$ let $j$ be such that $f_{j}: X \rightarrow I_{j}$; the projection of the inverse limit is a $\frac{\min (\varepsilon, \delta)}{10}$ mapping. Let $N>0$ be an integer such that $\operatorname{diam} f_{j}^{-1}\left(\left[\frac{i}{N}, \frac{i+1}{N}\right]\right) \leq \frac{\min (\varepsilon, \delta)}{5}$. Let $M$ be an integer such that for $m \geq M$ the Hausdorff distance between $f_{j}(K)$ and $f_{j}\left(K_{m}\right)$ is less than $\frac{1}{4 N}$. Now, let $f_{k}: X \rightarrow I_{k}, k>j$, be a projection such that the images $f_{k}\left(K_{M}\right), f_{k}\left(K_{M+1}\right), \ldots, f_{k}\left(K_{M+4 N+4}\right)$ are mutually disjoint and let $i_{0}<i_{0}+1<i_{1} \leq N$ be indices such that

$$
\inf \left(\rho(x, y): x \in f_{j}^{-1}\left(\frac{i_{0}}{N}\right), y \in f_{j}^{-1}\left(\frac{i_{1}}{N}\right)\right)>\delta \quad \text { and } \quad\left\{\frac{i_{0}}{N}, \frac{i_{1}}{N}\right\} \subseteq f_{j}\left(K_{m}\right),
$$

for every $m>M$. Now we define a sequence $a_{1} \leq b_{1}<c_{1} \leq d_{1}<a_{2} \cdots<a_{2 N} \leq$ $b_{2 N}<c_{2 N} \leq d_{2 N}<a_{2 N+1}$ of points of the segment $I_{k}$. We assume that the continua $K_{M}, K_{M+1}, \ldots, K_{M+4 N+4}$ are indexed in accordance with the order in $I_{k}$ of their images under the mapping $f_{k}$. The point $a_{1}$ is the first point of $I_{k}$ belonging 


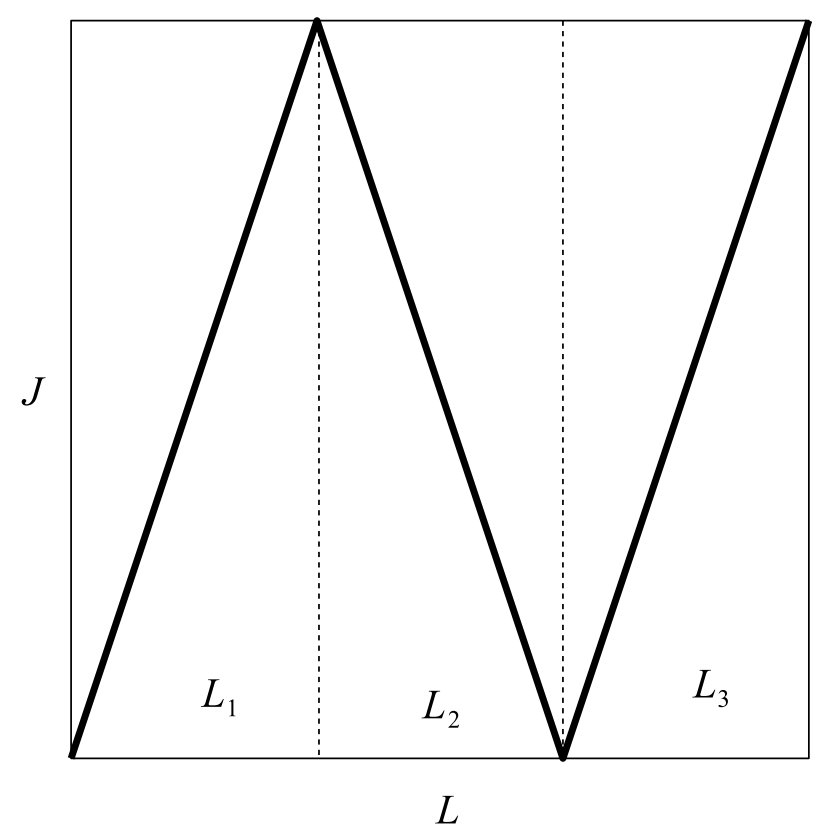

FiguRE 1

to $f_{k}\left(K_{M}\right)$ for which $f_{j}^{k}\left(a_{1}\right)=\frac{i_{1}}{N}$, the point $c_{1}$ is the first point following $a_{1}$ such that $f_{j}^{k}\left(c_{1}\right)=\frac{i_{0}}{N}, b_{1}$ is a point of absolute maximum of $f_{j}^{k}$ over $\left[a_{1}, c_{1}\right], a_{2}$ is the first point following $c_{1}$ such that $f_{j}^{k}\left(a_{2}\right)=\frac{i_{1}}{N}, d_{1}$ is the point of absolute minimum of $f_{j}^{k}$ over $\left[c_{1}, a_{2}\right]$, etc. After $n$ steps of the construction the arc $f_{k}\left(K_{M+2 n+1}\right)$ lies behind $\left[a_{1}, a_{n}\right]$, so we can continue this procedure. Let $n(x)$ for $x \in I_{k}$ be the integer fulfilling $f_{j}^{k}(x) \in\left(\frac{n(x)}{N}, \frac{n(x)+1}{N}\right]$. Each strictly monotone sequence of $n\left(b_{i}\right)$ is shorter than $N$, hence there is an index $l$ fulfilling $n\left(b_{l}\right) \geq n\left(b_{l+1}\right) \leq$ $n\left(b_{l+2}\right)$. We can assume that $f_{j}^{k}\left(d_{l}\right) \geq f_{j}^{k}\left(d_{l+1}\right)$ (otherwise we can invert the order of $\left.I_{k}\right)$. Let $q_{0}: I_{j} \rightarrow I_{j}$ be a nondecreasing surjection which maps the interval $\left[\frac{n\left(b_{l+1}\right)}{N}, \frac{n\left(b_{l+1}\right)+1}{N}\right]$ to a point, and this interval is the only fiber of this mapping different from a one-point set. Let $q=q_{0} f_{j}^{k}$. Let us remark that $q f_{k}$ is an $\varepsilon$ mapping and that $q\left(b_{l}\right) \geq q\left(b_{l+1}\right) \leq q\left(b_{l+2}\right)$ and $q\left(d_{l}\right) \geq q\left(d_{l+1}\right)$, and $q\left(b_{n}\right)>q\left(d_{m}\right)$ for all indices $n, m$. Now $d_{l}$ is a point of absolute minimum and $b_{l}$ is a point of absolute maximum of $q$ over $\left[a_{l}, a_{l+1}\right]$. Let us consider a nondecreasing sequence of five real numbers: $z_{1}=b_{l}, z_{2}=b_{l}+q\left(b_{l}\right)-q\left(d_{l}\right), z_{3}=z_{2}+q\left(b_{l+1}\right)-q\left(d_{l}\right), z_{4}=$ $z_{3}+q\left(b_{l+1}\right)-q\left(d_{l+1}\right), z_{5}=z_{4}+1-d_{l+1}$. We define mappings $p_{0}: I \rightarrow\left[0, z_{5}\right]$ and $r:\left[0, z_{5}\right] \rightarrow I$ by the following formulae:

$$
p_{0}(t)= \begin{cases}t, & \text { for } t \in\left[0, b_{l}\right), \\ z_{1}+q\left(b_{l}\right)-q(t), & \text { for } t \in\left[b_{l}, d_{l}\right), \\ z_{2}+q(t)-q\left(d_{l}\right), & \text { for } t \in\left[d_{l}, b_{l+1}\right), \\ z_{3}+q\left(b_{l+1}\right)-q(t), & \text { for } t \in\left[b_{l+1}, d_{l+1}\right), \\ z_{4}+t-d_{l+1}, & \text { for } t \in\left[d_{l+1}, 1\right]\end{cases}
$$


and

$$
r(z)= \begin{cases}q(z), & \text { for } z \in\left[0, z_{1}\right), \\ z_{1}+q\left(b_{l}\right)-z, & \text { for } z \in\left[z_{1}, z_{2}\right), \\ z-z_{2}+q\left(d_{l}\right), & \text { for } z \in\left[z_{2}, z_{3}\right), \\ z_{3}+q\left(b_{l+1}\right)-z, & \text { for } z \in\left[z_{3}, z_{4}\right), \\ q\left(z-z_{4}+d_{l+1}\right), & \text { for } z \in\left[z_{4}, z_{5}\right] .\end{cases}
$$

One can easily check that $q=r p_{0}$. Let $p=p_{0} f_{k}$. We can put $L_{1}=\left[z_{1}+q\left(b_{l}\right)-\right.$ $\left.q\left(b_{l+1}\right), z_{2}\right], L_{2}=\left[z_{2}, z_{3}\right], L_{3}=\left[z_{3}, z_{3}+q\left(b_{l+1}\right)-q\left(d_{l}\right)\right]$, and $L=L_{1} \cup L_{2} \cup L_{3}$, $T=\left[0, z_{5}\right], W=I_{j}$. Each mapping of a continuum onto an arc is weakly confluent [3, hence there exists a continuum $Y \subset X$, such that $p(Y)=L$.

Lemma 2. Let $f: X \rightarrow S$ be a mapping between compacta.

Assume that there exists a mapping $\nu: X \times X \rightarrow S$ fulfilling the following conditions: $\nu(x, y)=\nu(y, x)$ and $\nu(x, x)=f(x)$ for $x, y \in X$. Then the induced homomorphism between cohomology groups $f^{*}: H^{1}\left(S, \mathbb{Z}_{2}\right) \rightarrow H^{1}\left(X, \mathbb{Z}_{2}\right)$ must be zero.

Proof. We have the following commuting diagram:

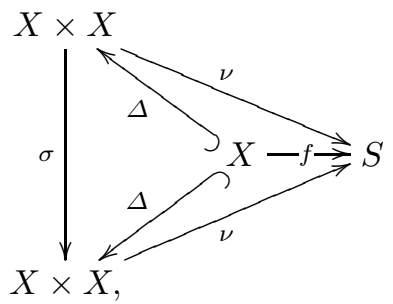

in which $\sigma: X \times X \rightarrow X \times X$ denotes the permutation homeomorphism defined by $\sigma((x, y))=(y, x)$ and $\Delta: X \rightarrow X \times X$ is given by $\Delta(x)=(x, x)$. It induces a commuting diagram of cohomology modules of the form

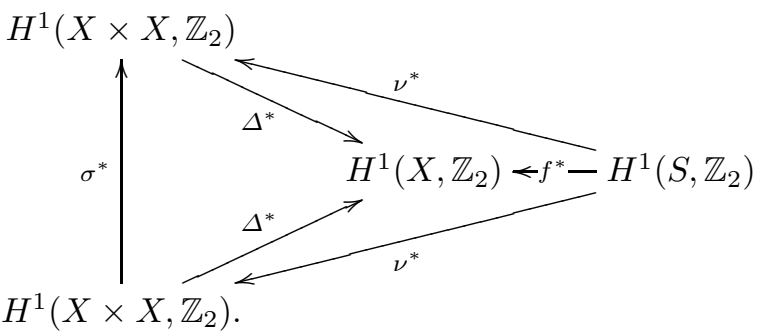

From the Künneth formula, taking into account that $\mathbb{Z}_{2}$ is a field, and hence any module over it is torsion free (cf. [7), we infer that $H^{1}\left(X \times X, \mathbb{Z}_{2}\right)$ is isomorphic to $H^{0}\left(X, \mathbb{Z}_{2}\right) \otimes H^{1}\left(X, \mathbb{Z}_{2}\right) \oplus H^{1}\left(X, \mathbb{Z}_{2}\right) \otimes H^{0}\left(X, \mathbb{Z}_{2}\right)$. For convenience we will identify these two objects. For an element $g \in H^{1}\left(S, \mathbb{Z}_{2}\right)$ we have a unique decomposition $\nu^{*}(g)=v+w$, where $v \in H^{0}\left(X, \mathbb{Z}_{2}\right) \otimes H^{1}\left(X, \mathbb{Z}_{2}\right)$ and $w \in H^{1}\left(X, \mathbb{Z}_{2}\right) \otimes H^{0}\left(X, \mathbb{Z}_{2}\right)$. The components $v$ and $w$ are sums of elements of the form $e_{0} \otimes e_{1}$ and $e_{1}^{\prime} \otimes e_{0}^{\prime}$, respectively, where $e_{0}, e_{0}^{\prime} \in H^{0}\left(X, \mathbb{Z}_{2}\right)$ and $e_{1}, e_{1}^{\prime} \in H^{1}\left(X, \mathbb{Z}_{2}\right)$. We have $\sigma^{*}\left(e_{0} \otimes\right.$ $\left.e_{1}\right)=e_{1} \otimes e_{0}$ and $\sigma^{*}\left(e_{1}^{\prime} \otimes e_{0}^{\prime}\right)=e_{0}^{\prime} \otimes e_{1}^{\prime}([9])$. This means that $\nu^{*}(g)=\sigma^{*} \nu^{*}(g)$ is a sum of elements of the form $e_{0} \otimes e_{1}+e_{1} \otimes e_{0}$. From the diagram $f^{*}(g)=\Delta^{*}\left(\nu^{*}(g)\right)$ and $\Delta^{*}\left(e_{0} \otimes e_{1}\right)+\Delta^{*}\left(e_{1} \otimes e_{0}\right)=\Delta^{*}\left(e_{0} \otimes e_{1}\right)+\Delta^{*}\left(\sigma^{*}\left(e_{1} \otimes e_{0}\right)\right)=\Delta^{*}\left(e_{0} \otimes e_{1}\right)+$ $\Delta^{*}\left(e_{0} \otimes e_{1}\right)=0$; hence $f^{*}$ is 0 . 


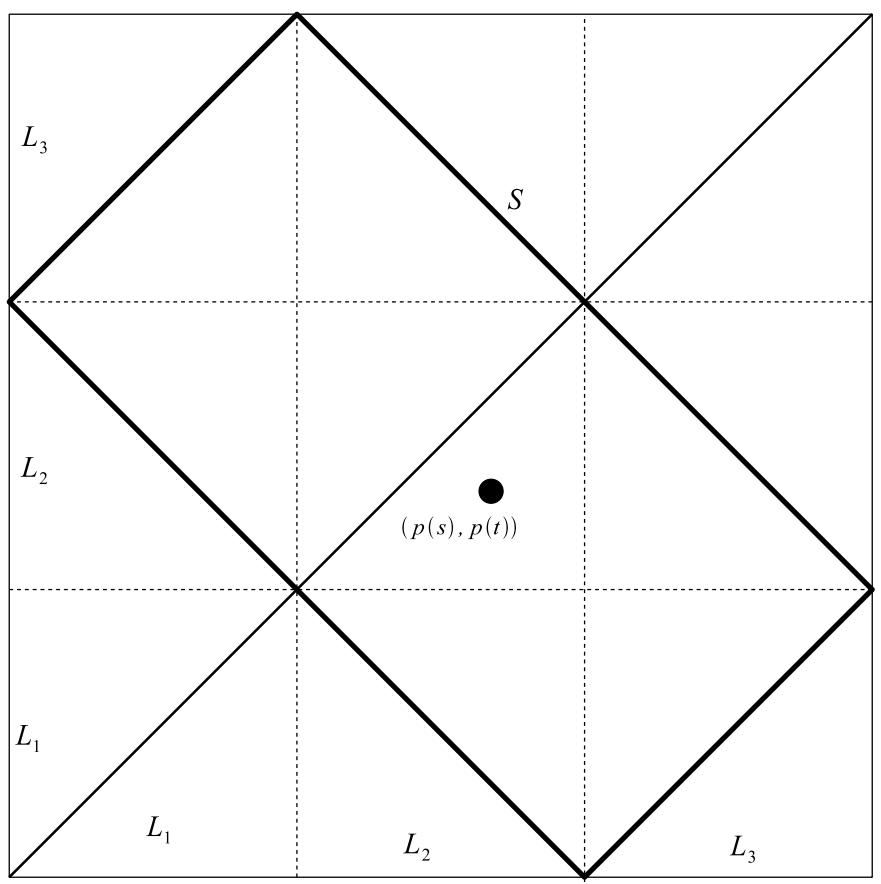

Figure 2. The set $G$.

Proposition 3. If $X$ is an acyclic continuum, and $a, b \in X$ and $f: X \rightarrow[0,1]$ is a mapping such that $f(a)=0, f(b)=1$, then the homomorphism $(f \times f)^{*}$ : $H^{*}\left(([0,1],\{0,1\}) \times\left([0,1],\{0,1\}, \mathbb{Z}_{2}\right)\right) \rightarrow H^{*}\left((X,\{a, b\}) \times(X,\{a, b\}), \mathbb{Z}_{2}\right)$ induced by the mapping $f \times f:(X,\{a, b\}) \times(X,\{a, b\}) \rightarrow([0,1],\{0,1\}) \times([0,1],\{0,1\})$ is an isomorphism.

Proof. First from the functoriality of the exact sequence for a closed pair and the Five Isomorphisms Lemma we infer that

$$
f^{*}: H^{*}\left(([0,1],\{0,1\}), \mathbb{Z}_{2}\right) \rightarrow H^{*}\left((X,\{a, b\}), \mathbb{Z}_{2}\right)
$$

is an isomorphism. Then we apply the same reasoning to the relative Künneth formula for $(f \times f)^{*}([9]$, p. 360).

Theorem 2. If a chainable continuum $X$ admits a mean, then $X$ is an arc.

Proof. Suppose $X$ is a chainable continuum which is not an arc and $\mu: X \times X \rightarrow X$ is a mean. Let $\delta>0$ be such as in the Long Fold Lemma, Lemma 11 and let $\eta=0.1 \delta$. From Proposition 2 we have a number $\varepsilon>0$, smaller than $\eta$ such that $\mu_{X \times X}\left(\Delta_{\varepsilon} \times \Delta_{\varepsilon}\right) \subset \Delta_{\eta}$. Now let mappings $p: X \rightarrow T, r: T \rightarrow W$, where $T, W$ are arcs, and a subcontinuum $Y \subset X$ satisfies conditions (i), (ii), (iii) and (iv) of the Long Fold Lemma. Because $\operatorname{diam} Y>\delta$ and $r p$ is an $\varepsilon$-mapping, there exist points $s, t \in Y$ such that $p(s), p(t) \in L_{2}$, where $L_{1}, L_{2}, L_{3}$ are such as in condition (iv) of the lemma, and $\rho(s, t)>0.5 \delta$; thus $(p(s), p(t)) \notin p \times p\left(\Delta_{\eta}\right)$. Let us consider the set $G=\{(x, y) \in L \times L: r(x)=r(y)\}$. (See Figure 2.) It contains a simple closed curve $S$ surrounding $(p(s), p(t))$ (in the figure it is drawn with a thick line). Let $d: T \times T \backslash\{(p(s), p(t))\} \rightarrow S$ be a retraction. Denote $S^{\prime}=(p \times p)^{-1}(S)$. 
If $\left(x_{1}, x_{2}\right) \in S^{\prime} \subset X \times X$, then $r p\left(x_{1}\right)=\operatorname{rp}\left(x_{2}\right)$; consequently $S^{\prime} \subset \Delta_{\varepsilon}$. Define $\nu: S^{\prime} \times S^{\prime} \rightarrow S$ by the formula $\nu(x, y)=d\left(p \times p\left(\mu_{X \times X}(x, y)\right)\right)$, for $x, y \in S^{\prime}$. We have $\nu(x, y)=\nu(y, x)$ and $\nu(x, x)=p \times p(x)$; hence by Lemma 2 the induced homomorphism $\left(p \times p \mid S^{\prime}\right)^{*}: H^{1}\left(S, \mathbb{Z}_{2}\right) \rightarrow H^{1}\left(S^{\prime}, \mathbb{Z}_{2}\right)$ must be zero. But on the other hand consider the diagram of homomorphisms induced by appropriate restrictions of the mapping $p \times p$ between two cohomology Mayer-Vietoris sequences for excisive pairs. One of them is the sequence for pairs $(D, \emptyset),(E, K),(Q, K)=(D, \emptyset) \cup(E, K)$, where $D$ is the "rectangle" bounded by $S, E=\operatorname{cl}(T \times T \backslash D), a, b$ are the ends of $T, K=\{a, b\} \times T \cup T \times\{a, b\}$, and $(Q, K)=(T,\{a, b\}) \times(T,\{a, b\})$. The second is the sequence for pairs $\left(D^{\prime}, \emptyset\right),\left(E^{\prime}, K^{\prime}\right),\left(Q^{\prime}, K^{\prime}\right)=\left(D^{\prime}, \emptyset\right) \cup\left(E^{\prime}, K^{\prime}\right)$, where $D^{\prime}, E^{\prime}$ are inverse images under $p \times p$ of $D, E$, respectively, $a^{\prime}, b^{\prime} \in X$ are such that $p\left(a^{\prime}\right)=a, p\left(b^{\prime}\right)=b$ and $\left(Q^{\prime}, K^{\prime}\right)=\left(X,\left\{a^{\prime}, b^{\prime}\right\}\right) \times\left(X,\left\{a^{\prime}, b^{\prime}\right\}\right)$. Remark that $S=D \cap E$ and $S^{\prime}=D^{\prime} \cap E^{\prime}$. Let us write the following fragment of this diagram:

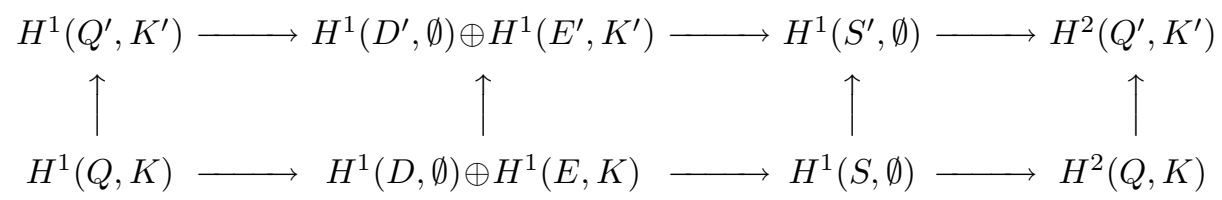

(for brevity we omitted the ring of coefficients, which is $\mathbb{Z}_{2}$ ). From Proposition 3 the last vertical arrow represents an isomorphism, and the direct sum in the lower row is zero ( $D$ is contractible and the pair $(E, K)$ is homotopically equivalent to the pair $(K, K))$. From exactness of the lower row and commutativity of the diagram the homomorphism from $H^{1}\left(S, \mathbb{Z}_{2}\right)$ to $H^{1}\left(S^{\prime}, \mathbb{Z}_{2}\right)$ must be nonzero, a contradiction.

Example 1. Another question Bacon asked in 2] is the following: Is the arc the only continuum containing an open dense ray (i.e., a subspace homeomorphic to the half-line $[0, \infty))$ that admits a mean? The space $\Sigma^{\prime}$ described below may serve as a counterexample to this question. The diadic solenoid $\Sigma$ as a topological group admits a $1-1$ homomorphism $\varphi: \mathbb{R} \rightarrow \Sigma$ of the group of real numbers onto the composant of the neutral element in $\Sigma$. As was remarked by Sigmon in 7] (cf. [5]), solenoid $\Sigma$ has the unique division by 2. Let us define $\Sigma^{\prime}=\Sigma \times\{0\} \cup\left\{\left(\varphi(t), \frac{1}{t+1}\right)\right.$ : $t \in[0, \infty)\} \subset \Sigma \times \mathbb{R}$. Now we can define a mean on $\Sigma^{\prime}$ by the following formulae:

i) $\mu\left(\left(x, z_{1}\right),\left(y, z_{2}\right)\right)=\left(\frac{x+y}{2}, 0\right)$ if $z_{1}=0$ or $z_{2}=0$ and $x, y \in \Sigma$;

ii) $\mu\left(\left(\varphi\left(t_{1}\right), \frac{1}{t_{1}+1}\right),\left(\varphi\left(t_{2}\right), \frac{1}{t_{2}+1}\right)\right)=\left(\varphi\left(\frac{t_{1}+t_{2}}{2}\right), \frac{1}{\frac{t_{1}+t_{2}}{2}+1}\right)$ for $t_{1}, t_{2} \in[0, \infty)$.

Remark 1. A generalization of means are $n$-means.

Definition 3. Let $X$ be a space and $n$ an integer, $n \geq 2$. A mapping $\mu: X^{n} \rightarrow X$ is called an $n$-mean if $\mu\left(x_{\sigma(1)}, \ldots, x_{\sigma(n)}\right)=\mu\left(x_{1}, \ldots, x_{n}\right)$ for every $x_{1}, \ldots, x_{n} \in X$ and each permutation $\sigma$ of indices $1, \ldots, n$, and $\mu(x, \ldots, x)=x$ for every $x \in X$.

A slight modification of the presented argument of Theorem 2 (as a ring of coefficients of cohomology we should use $\mathbb{Z}_{p}$ instead of $\mathbb{Z}_{2}$ ) allows us to show that for any prime integer $p$ the only chainable continuum admitting a $p$-mean is the arc. But, as was observed by Sigmon [7, if a space admits an $n$-mean, then it admits a $p$-mean for each prime divisor $p$ of the integer $n$. Hence we have the following general theorem.

Theorem 3. Let $n \geq 2$ be an integer. A chainable continuum $X$ admits an $n$-mean if and only if $X$ is an arc (cf. [1]). 


\section{REFERENCES}

1. M.M. Awartani, D.W. Henderson, Compactifications of the ray with the arc as remainder admit no n-mean, Proc. Amer. Math. Soc. 123 (1995), no. 10, pp. 3213-3217. MR1307490 (95m:54027)

2. P. Bacon, An acyclic continuum that admits no mean, Fund. Math. 67 (1970), pp. 11-13. MR0261555(41:6168)

3. R. H. Bing, Snake-like continua, Duke Math. J. 18 (1951), pp. 653-663. MR0043450 (13:265a)

4. J.J. Charatonik, Means on arc-like continua, an essay in Open Problems in Continuum Theory by W. Charatonik and J. Prajs on http://web.umr.edu/ continua/

5. P. Krupski, Means on solenoids, Proc. Amer. Math. Soc. 131 (2003), no. 6, pp. 1931-1933 (electronic). MR $1955283(2003 \mathrm{j}: 54028)$

6. K. Kuratowski, Topology II, Mir (1969) (in Russian). MR0259836 (41:4468)

7. K. Sigmon, Acyclicity of compact means, Michigan Math. J. 16 (1969), pp. 111-115. MR0259899 (41:4528)

8. M. Sobolewski, Pseudo-contractibility of chainable continua, Topology Appl. 154 (2007), no. 16, 2983-2987. MR 2355883

9. E.H. Spanier, Algebraic topology, McGraw-Hill, New York (1966). MR0210112 (35:1007)

Instytut Matematyki, Banacha 2, Warszawa 02-097, Poland

E-mail address: msobol@mimuw.edu.pl 